\title{
Untersuchungen an Kurzwellen-Echosignalen
}

\author{
Von Hanns Albrecht Hess*
}

(Z. Naturforschg. 1, 499-505 [1946]; eingegangen am 5. Juni 1946)

\begin{abstract}
Eine Meßmethode hoher Genauigkeit wurde für Zeichenuntersuchungen bei Telegraphie-Kurzwellensendern entwickelt. Uberraschend genaue Laufzeitmessungen an Echosignalen entfernter Kurzwellensender im Frequenzbereich zwischen 10 und $20 \mathrm{MHz}$ gelangen unter bestimmten ionosphärischen Bedingungen zu wenigen täglichen Stunden. Die Umlaufzeit eines Signals um den Erdball ergab sich als ein konstanter Wert zu 0,137788 Sekunden. Dieser scheint unabhängig von der Frequenz, Tageszeit und Jahreszeit zu sein. Entfernungsbestimmungen bei mehr als $1000 \mathrm{~km}$ vom Beobachtungsort entfernten Kurzwellensendern ließen sich mit einer Genauigkeit von $\pm 25 \mathrm{~km}$ durchführen. Diese aus zahlreichen Messungen der Jahre 1941-1944 gewonnenen Tatsachen ferner Amplitudenvergleiche bei Signalen, die mehrmals den Erdball umkreist hatten, sprechen für die v.Schmidtsche Theorie einer sogen. Gleitwellen-Ausbreitung entlang einer ionosphärischen Grenzschicht zwischen zwei verschiedenen Medien und gegen die bisherige Anschauung einer Kurzwellenübertragung, deren Zustandekommen durch mehrfache Zickzack-Reflexionen zwischen Ionosphäre und Erdoberfläche angenommen wird.
\end{abstract}

$\mathrm{D}_{\mathrm{l}}^{\mathrm{a}}$ as Auftreten der mehrfachen Zeichen bei der Kurzwellen-Ausbreitung ist im Schrifttum seit dem Jahre 1926 bekannt. Untersuchungen auf diesem Gebiet wurden von $Q u a ̈ c k$ und $M \ddot{o}$ gel (Deutschland) ${ }^{1}$, T. L. Eckersley (England $)^{2}$ und A. H. T a y lo r und L. C. Y o ung (USA.) ${ }^{3}$ durchgeführt. M ögel fand für die gemessenen Laufzeiten der Echosignale Werte, die nur um $1 \%$ verschieden waren. Taylor und Y oung fanden größere Abweichungen der Laufzeiten voneinander, und zwar in der Größenordnung von $5 \%$. Da solche Ergebnisse in einem bemerkenswerten Widerspruch zu den Echolotungen an den ionosphärischen Schichten standen, wurde besonders die Richtigkeit der $\mathrm{M}$ ö gelschen Messungen angezweifelt, denn bekanntlich sind die bei der Echolotung gemessenen Höhen der Ionosphärenschichten durch starke tägliche und jahreszeitliche Schwankungen gekennzeichnet.

O. v. Schmidt hat auf Grund der Mögelschen Messungen eine neue Theorie der Kurzwellen-Ausbreitung entwickelt. An dem von ihm geleiteten Institut für Physik in Berlin-Gatow wurde 1941 mit diesbezüglichen Messungen begonnen. Es sollte durch systematische Untersuchungen mit verbesserten Geräten festgestellt

* Ulm (Donau), Schadstr. 24.

1 E. Quäck, Neues üher die Ausbreitung von kurzen Wellen, Jb. drahtl. Tel. 28, 177 [1926]; E. Qu ä c k, Weitere Mitteilungen ïber die Ausbreitung von Kurzwellen, Jb. drahtl. Tel. 30, 41 [1927]; Q ü̈ck u. Mögel, Doppel- und Mehrfachzeichen bei Kurzwellen, E.N.T. 6, 45 [1929]. werden, ob die Erdumläufe durch mehrfache Zickzack-Reflexionen zwischen den ionosphärischen Schichten und der Erdoberfläche, oder durch eine Gleitwellen-Ausbreitung entlang einer ioñosphärischen Grenzschicht erklärt werden könnten.

Die ersten in Berlin-Gatow im September und Oktober 1941 mit erheblich verbesserten Apparaten durchgeführten Messungen zeigten bei Kurzwellensendern aus Europa, Ostasien und Südamerika überraschende Ergebnisse, so daß sich bei mehr als $1000 \mathrm{~km}$ entfernten Stationen eine genaue Bestimmung der Entfernung als möglich erwies.

Da in Ländern höherer geographischer Breite auf Grund einfacher Überlegungen eine über längere tägliche Zeiten dauernde Echotätigkeit bei Kurzwellen-Signalen erwartet werden mußte, wurde zum Zwecke gründlicher Untersuchungen eine wissenschaftliche Expedition nach Dänemark ausgerüstet. Vom 6. Dez. 1941 bis 1. April 1944 wurden die Messungen in Frederikshavn - geographische Koordinaten: $57^{\circ} 26^{\prime} \mathrm{N}, 10^{\circ} 29^{\prime} \mathrm{E}-$ durchgeführt, und am 2. April 1944 wurde die Station nach Randers - geographische Koor-

2 Short wave wireless telegraphy, Journal I.E.E. 600 [1927].

3 Studies of High-Frequency Radio-Wave-Propagation Proc. I.R.E. 16, Nr. 5 [1928].

4 Uber die Totalreflexion in der Akustik und Optik, Ann. Physik 19, 891 [1934]. Neue Erklärung des Kurzwellenumlaufes um die Erde, Z. Techn. Physik 17, 443 [1936]. 
dinaten: $56^{0} 31^{\prime} \mathrm{N}, 10^{\circ} 02^{\prime} \mathrm{E}$ - verlegt. Dort verblieb die Expedition bis zu ihrer Auflösung im Januar 1945.

\section{Technischer Teil}

In Abb. 1 ist die Zusammenstellung der Geräte wiedergegeben, mit denen in Frederikshavn die Messungen durchgeführt wurden. $E$ ist ein normaler Kurzwellen-Superhet-Empfänger (Philips CR 101). Die $\mathrm{Z}$ wischenfrequenz dieses Empfängers, die etwa bei $750 \mathrm{KHz}$ liegt, wurde mittels eines dazwischengeschalteten kleinen Kondensators auf die Mischstufe $M$ geleitet. Durch einen variablen Oszillator wurde diese $\mathrm{Z}$ wischenfrequenz überlagert und es ent-

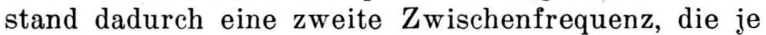
nach der Art der photographischen Aufnahmen zwischen 50 und $100 \mathrm{KHz}$ verändert werden konnte. Diese zweite $\mathrm{Z}_{w}$ ischenfrequenz, die die empfangenen Funksignale übertrug, wurde auf den einen Strahl des

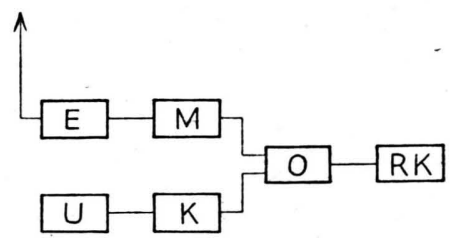

Abb. 1. Schema der experimentellen Anordnung.

Zwei-Strahl-Oszillographen $O$ geleitet. Sie wurde "Schreibfrequenz" genannt, da sie für die photographische Wiedergabe der Funksignale auf den Filmstreifen verantwortlich war. Auf den anderen Strahl des Oszillographen wurde eine $500-\mathrm{Hertz}$ - Meßfrequenz gebracht, die durch einen $500-\mathrm{Hertz}$-Stimmgabelgenerator $U$ erzeugt wurde. Durch einen Thermostaten konnte die Meßfrequenz auf $10^{-7} \mathrm{Hertz}$ konstant gehalten werden. Die sinusförmige $500-\mathrm{He}$ r z-Frequenz war für genaue Messungen ungeeignet und wurde daher in einem. Kippgerät $K$ verzerrt. Die auf diese Weise erhaltenen Zacken mit einem Abstand von 2 Millisekunden gestatteten eine sichere Vermessung durch das Mikroskop bei einer Genauigkeit von 1/100 mm. Durch die Registrierkamera $R K$ werden die Funksignale und die Meßfrequenzzacken auf die rasch ablaufenden Papierfilmstreifen aufgenommen. Die Filme hatten im allgemeinen eine Länge von $10 \mathrm{~m}$.

Bei Messungen in Berlin-Gatow wurde die 1000Hertz-Normalfrequenz der Physikalischen Technischen Reichsanstalt benutzt, die durch ein besonderes Kabel dorthin übertragen wurde. Die Quarzfrequenz der PTR hatte eine Genauigkeit von 10-9 Hertz.

Für den Kurzwellenempfang wurden Richtantennen benutzt. Wegen ihrer Strahlungscharakteristik schienen die sogen. „Langdrahtantennen“ am besten für die Messungen geeignet zu sein, denn solche Systeme, die mehrere aneinandergereihte Dipole darstellen, strahlen bzw. empfangen gebündelt aus zwei entgegengesetzten Richtungen. Diese sind nahezu durch die gerade Richtung des Antennendrahtes als Achse festgelegt. Für den Empfang des direkten und des indirekten Zeichens, letzteres kommt gerade aus der entgegengesetzten Richtung an, stellen solche Antennensysteme eine ausgezeichnete und billige Lösung dar. 4 Langdrahtantennen, jede mit einer Länge von etwa $100 \mathrm{~m}$ ( $5 \lambda$ bei $20 \mathrm{~m}$ Wellenlänge) wurden in Frederikshavn für die folgenden 4 Himmelsrichtungen benutzt: 1. Nord-Süd, 2. Nordost-Südwest, 3. West-Ost, 4. Nordwest-Südost.

Die Geräte zur Aufnahme der Kurzwellensignale wurden in bezug auf ihre Melgenauigkeit und die photographische Wiedergabe gegenüber denen erhel,lich verbessert, die durch $M$ ögel in den Jahren 1927 bis 1934 benutzt worden waren. Folgende wesentliche Verbesserungen wurden erzielt:

1. Die Frequenz, die auf das Braunsche Rohr des Oszillographen gegeben wurde und die für die Zeichnung der Kurzwellensignale auf den Filmstreifen verantwortlich war, wurde auf 50 bis $100 \mathrm{KHz}$ erhöht. M ögel benutzte damals eine Frequenz von $1000 \mathrm{Hertz}$, die er auf einen Schleifenoszillographen brachte.

2. Die Ablaufgeschwindigkeit der Papierfilmstreifen wurde von $40 \mathrm{~cm} / \mathrm{sec}$ auf $400 \mathrm{bis} 1000 \mathrm{~cm} / \mathrm{sec}$ erhöht.

3. Die Genauigkeit der Zeit-Meßfrequenz wurde von $1 \%$ auf $1 / 100 \%$ erhöht.

2. Ergebnisse der Messungen und Unter-

\section{a) Bestimmung der Zeiten der Echotätigkeit durch} systematische Beobachtungen

Schon die ersten Untersuchungen von $Q \mathbf{u} a ̈ c k$ und Mögel während der Jahre 1926 bis 1933 zeigten, daß das Auftreten und die Intensität der rückwärtigen Zeichen und Erdumläufe von der Tageszeit und der Jahreszeit abhängen. Auch Veränderungen, die sich über mehrere Jahre erstreckten und im Zusammenhang mit der elfjährigen Sonnenfleckenperiode stehen, wurden damals schon erkannt. Diese Autoren gelangten auf Grund ihrer Beohachtungen zu der Erkenntnis, daß die eine normale Kurzwellenübertragung allgemein stark beeinträchtigenden rückwärtigen Zeichen und Erdumläufe fast ausschließlich während der Dämmerungszeit auftreten. Als Zone für eine optimale Aushreitung solcher Echosignale wurde der den Erdball umgebende Dämmerungsgürtel angesehen.

Alle diese früheren Feststellungen haben sich bei den neueren Untersuchungen ebenfalls wieder ergeben. Jedoch mußten auch zuweilen größere Abweichungen vom Dämmerungsgroßkreis hinsichtlich des Auftretens der Echosignale verzeichnet werden. Systematische, üher ein Jahr in Frederikshavn durchgeführte Untersuchungen lieferten üher das zeitliche Auftreten der Echosignale sowie über ihre Stärke und besonders über die Art und Kennzeichen derselhen umfangreiches Material. Abh. 2 zeigt bei Kurzwellensendern in USA., Japan und Südamerika die im Verlauf von 12 Monaten gewonnenen Aufzeich- 
nungen über die Echos zu den einzelnen Tagesstunden. Da jeden halben Monat eine Zusammenstellung gemacht wurde, sind 24 Diagramme für 24 halbe Monate eingetragen. Für jedes Diagramm ist auf der Abszisse die Uhrzeit (MEZ.) aufgetragen; die Ordinate beurteilt die relative Stärke der Echosignale, geschätzt entsprechend den funkerischen Gepflogenheiten des Fram-Codes nach Lautstärke ,1-5“. Die eingetragenen Kurven für die Echolautstärke stellen das Mittel von je 15 Tagen dar. Aus der Abbildung ist $\mathrm{zu}$ ersehen, daß die Echotätigkeit $\mathrm{zu}$ verschiedenen Tageszeiten auftritt, und man kann erkennen, daß hierbei die jeweilige Richtung des Dämmerungsgür-
Bei Stationen in Argentinien und Brasilien wurden dagegen ausnahmslos nur die direkten Zeichen und die Erdumläufe derselben festgestellt. Bei Abendaufnahmen an japanischen Stationen trat sehr häufig die Eigentümlichkeit in Erscheinung, daß die Feldstärke des rückwärtigen Zeichens oftmals größer war als die des direkten Zeichens.

Ob diese seltsamen Erscheinungen tatsächlich auf ionosphärischen Gesetzmäßigkeiten beruhen, oder ob rein technische Dinge, wie beispielsweise „Richtantennen“ bei den Kurzwellensendern, als Ursache hierfür anzusprechen sind, konnte durch die Untersuchungen nicht geklärt werden.

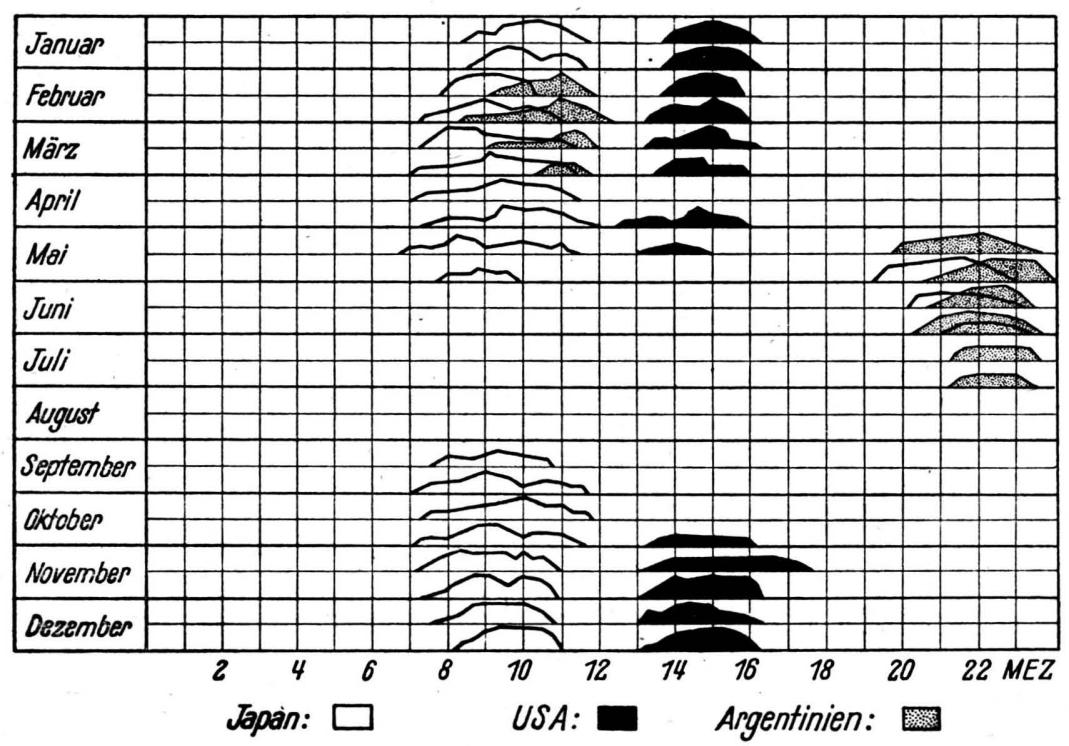

Abb. 2. Echotätigkeit in ihrer Abhängigkeit von den Jahres- und Tageszeiten.

tels maßgebend ist. So treten Echos während des Winterhalbjahres bei Ostasien und Südamerika stets. vormittags und bei Nordamerika stets nachmittags in Erscheinung. Im Mai, Juni, Juli und August ereignete sich die Echotätigkeit bei Stationen aus Ostasien und Südamerika nur noch während einiger Nachtstunden. Im Mai allerdings bestand zur Übergangszeit bei Ostasien sowohl vormittags als auch abends Echotätigkeit.

Um frühere Ergebnisse von Mögel zu überprüfen, in denen zum Ausdruck kam, daß während der Nachtstunden des Sommerhalbjahres niemals rückwärtige Zeichen aufgetreten, sondern als Echosignale stets nur Erdumläufe festgestellt worden seien, wurden während der Nachtstunden der Monate Mai, Juni, Juli und August 1943 . Zeichenaufnahmen bei Kurzwellenstationen in Ostasien und Südamerika durchgeführt. Die Untersuchungen ergaben bei mehr als 80 Einzelfällen, daß bei japanischen Stationen während der Sommernacht stets nur rückwärtige Echosignale $\mathrm{zu}$ verzeichnen waren und daß nur in zwei Fällen außer den rückwärtigen Zeichen auch die Erdumläufe des direkten Zeichens vorhanden waren.

\section{b) Messungen an Funksignalen bei kommerziellen Telegraphie-Kurzwellensendern}

Das Funksignal eines Kurzwellensenders im Frequenzbereich zwischen 10 und $20 \mathrm{MHz}$ kann auf zwei Wegen vom Sender zum Empfänger gelangen, entweder auf dem direkten Großkreisweg, dann wird es in der vorliegenden Abhandlung direktes Zeichen benannt, oder das Zeichen gelangt auf dem gleichen Großkreis, aber in entgegengesetzter Richtung, zum Empfänger, dann nennt man es indirektes oder rückläufiges Zeichen. Außer diesen gibt es noch Umläufe. Falls das direkte Zeichen noch vollständig um die Erde herumläuft, wird es direkter Umlauf genannt. Läuft dagegen das indirekte Zeichen noch um die Erde herum, dann nennt man es rückwärtigen Umlauf. Es gibt ferner Fälle, jedoch treten sie seltener auf und setzen besonders gute Empfangsverhältnisse voraus, in denen ein Zeichen zwei- und dreimal um die Erde herumläuft.

Es läßt sich leicht eine Formel ableiten, nach der aus Laufzeitmessungen an Kurzwellensignalen eine 
genaue Entfernungsbestimmung entfernter Kurzwellensender möglich ist. Sie lautet:

$$
\frac{t_{u}-t_{i}}{t_{u}}=\frac{2 d}{u}
$$

wo $t_{u}=$ Umlaufszeit (meßbar als Zeit zwischen dem Eintreffen des direkten Zeichens und des einmal direkt umgelaufenen Zeichens beim Empfangsort), $t_{i}=$ Zeit zwischen dem Eintreffen des direkten und des rückläufigen Zeichens, $d=$ Abstand Sender-Empfänger auf der Erdoberfläche, $u=$ Erdumfang.

Setzt man $u=40024 \mathrm{~km}$ und $t_{u}=0,137788 \mathrm{sec}$, so ergibt sich:

$$
d=\frac{40024}{2}\left(1-\frac{t_{i}}{0,137788}\right) \mathrm{km} .
$$

den mußte, noch sich zu irgendwelchen Tages- oder Jahreszeiten verändert hat. Ebenso haben Untersuchungen, ob der $t_{u}$-Wert bei Echosignalen aus der Ost-West-Richtung gegenüber solchen aus der Nord-Süd-Richtung irgendwelche Abweichungen erkennen ließ, noch zu keinem - wohl außerhalb der Meßgenauigkeit liegenden - Ergebnis geführt.

\section{d) Aufnahmen von rückläufigen Zeichen}

Die Filmaufnahmen mit direkten und indirekten Kurzwellensignalen ermöglichten eine Entfernungsbestimmung entfernter Sender mit überraschender Genauigkeit. In der oben abgeleiteten Formel ist dabei lediglich der Wert der im Filmstreifen gemessenen Laufzeitdifferenz $t_{i}$ zwischen dem direkten und dem rückläufigen Zeichen einzusetzen.

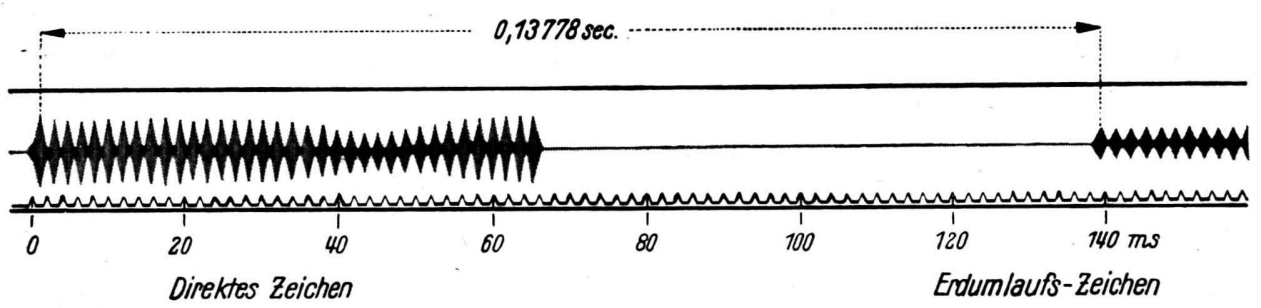

Abb. 3. Zeichen von LQB 2 - Monte Grande, Argentinien, 17570 kHz.

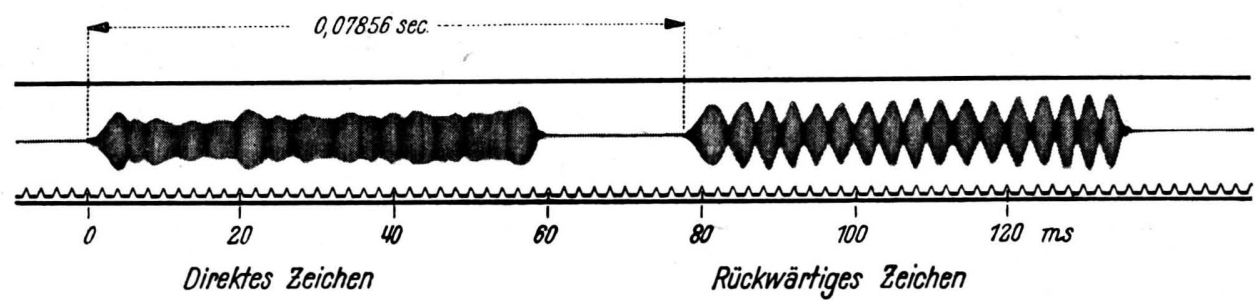

Abb. 4. Zeichen von JUP/JUD - Tokyo-Oyama, Japan, 13065 kHz, Entfernung 8598 km.

\section{c) Aufnahmen von Umlaufszeichen um die Erde}

Läuft ein direktes Zeichen in seiner ursprünglichen Richtung um die Erde herum, dann trifft es nach der Laufzeit $t_{\boldsymbol{u}}=0,137788$ sec zum zweitenmal beim Empfänger ein. Dieser Wert stellt das arithmetische Mittel aus 218 gut vermeßbaren Umlaufswerten dar.

Die Abb. 3 zeigt die Filmstreifenaufnahme von direktem Zeichen und direktem Umlauf bei der südamerikanischen Station LQB 2, Monte Grande, Argentinien, auf $17570 \mathrm{KHz}$. Der Sender ist stark durchmoduliert, wodurch eine genaue Vermessung der Laufzeiten wesentlich erleichtert wird.

Umfangreiche Untersuchungen haben ergeben, daß die Laufzeit $t_{u}$ eine Konstante ist. Die Meßgenauigkeit hätte Abweichungen noch in der 5. Dezimalstelle erkennen lassen. Seltsamerweise hat es sich gezeigt, daß der $t_{u}$-Wert in keinem Falle von der Frequenz des Senders abhängig ist, wie zunächst vermutet wer-
Die große Mehrzahl aller in Frederikshavn aufgenommenen Fiłmrollen zeigt direkte und rückläufige Zeichen. Es waren allein 47 verschiedene Kurzwellenstationen auf allen Erdteilen Gegenstand dieser Messungen. Die Messungen schließen Entfernungen zwischen 2000 und $17000 \mathrm{~km}$ ein. Die Frequenzen der empfangenen Stationen lagen im Bereich zwischen 10 und $19 \mathrm{MHz}$.

Abb. 4 zeigt Signale von der japanischen Station JUP/JUD-Tokyo-Oyama auf $13065 \mathrm{KHz}$. Es fällt dabei wohl besonders auf, daß das rückwärtige Zeichen mit etwas größerer Amplitude ankommt und auch schärfer gezeichnet ist, während beim direkten Zeichen die Modulation durch ein sogen. „Mehrweg-Phänomen" verzerrt wird, d.h. das direkte Zeichen wird durch nahezu gleichzeitig, aber mit verschiedenen Phasen ankommende Signale geformt.

Abb. 5 stellt eine Aufnahme bei der nordamerikanischen Station WQL-New Brunswick (USA.) auf $14815 \mathrm{KHz}$ dar. Tab. 1 zeigt die Vermessungswerte 


\begin{tabular}{|c|c|c|}
\hline $\begin{array}{c}\text { Laufzeit } t_{i} \\
\text { sec }\end{array}$ & $\begin{array}{c}\text { Gemessene } \\
\text { Entfernung km }\end{array}$ & $\begin{array}{c}\text { Fehler } \\
\mathrm{km}\end{array}$ \\
\hline 0.096000 & 6068 & +16 \\
0.096158 & 6045 & -7 \\
0.096814 & 6091 & +39 \\
0.096154 & 6046 & -6 \\
0.096080 & 6057 & +5 \\
\hline
\end{tabular}

Tab. 1. Entfernungsmessung an einer $6052 \mathrm{~km}$ entfernten nordamerikanischen Station am 12. März 1942 um $14^{\mathrm{h}} 40 \mathrm{MEZ}$.

eines etwa $10 \mathrm{~m}$ langen Filmstreifens dieser Station, auf dem sich fünf gut vermeßbare Stellen befinden.

\section{e) Messungen an Mehrfachumläufen}

Zum Zweck der genaueren Untersuchung von mehrfachen Erdumläufen wurden über die Kurzwellensender DLO - $19947 \mathrm{KHz}, \mathrm{DLN}-17670 \mathrm{KHz}$, DLK - $15075 \mathrm{KHz}$ und DLJ - $13925 \mathrm{KHz}$ der Deutschen Reichspost Punktsignale in größeren zeitlichen Abständen gesandt.

Besonders gute Ergebnisse über solche mehrfachen Umläufe erbrachten die in Randers (Dänemark) durchgeführten Aufnahmen vom 19. Nov. 1944, 09h00 bis $10^{\mathrm{h}} 00 \mathrm{MEZ}$, bei der Kurzwellenstation DLO-Rehmate auf $19947 \mathrm{KHz}$. Es sei dabei erwähnt, daß dieser Sender eine Richtantenne nach Japan benutzte, wodurch die rückwärtigen Zeichen unterdrückt wurden.

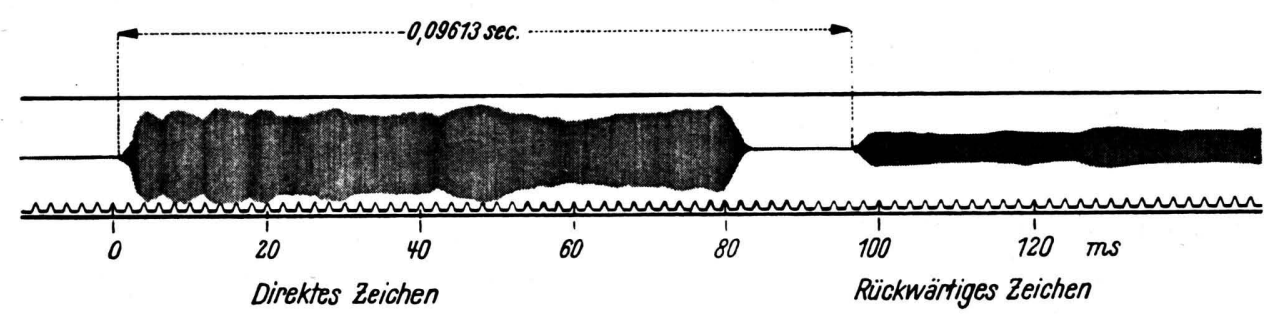

Abb. 5. Zeichen von WQL - New Brunswick, USA., $14815 \mathrm{kHz}$, Entfernung $6052 \mathrm{~km}$.

Direktes

Zeichen

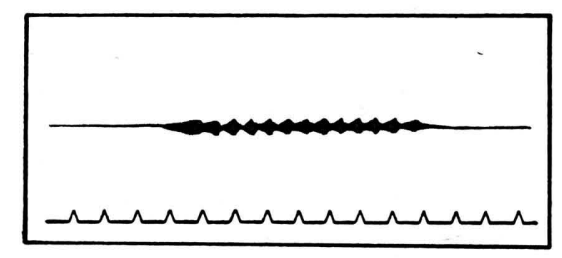

Erster

Erdumlauf

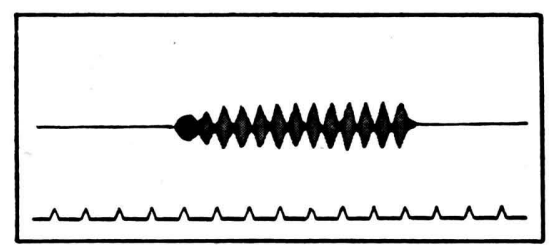

Zweiter

Erdumlauf

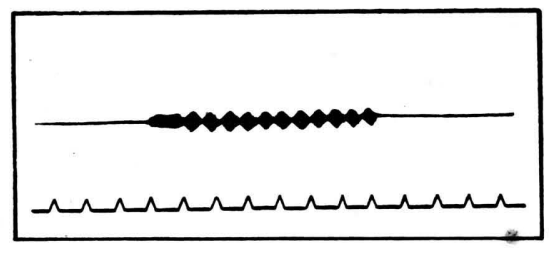

Dritter

Erdumlauf

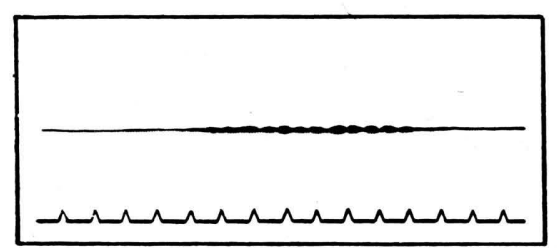

Abb. 6. Mehrfach-Umläufe um die Erde.
In Abb. 6 ist eine Filmaufnahme dieser mehrfachen Echos wiedergegeben. Die einzelnen Zeichen sind untereinander angeordnet, um so am besten verglichen werden zu können. Das Hauptzeichen, das bei nahen Stationen in mehrere, meist innerhalb kürzerer Laufzeiten nacheinander ankommende Einzelsignale zerlegt werden kann, zeigt nur geringe. Feldstärke. (Diese Beobachtung wurde in der Regel bei allen Kurzwellensendern gemacht, die weniger als $1000 \mathrm{~km}$ vom Empfänger entfernt sind. Eine Entfernungsbestimmung gelang in solchen Fällen nur ganz selten.) Am stärksten ist der erste Umlauf. Der zweite Umlauf zeigt etwa noch ein Drittel der Feldstärke des ersten Umlaufes, und ebenfalls zeigt der dritte Umlauf ein Drittel der Feldstärke des zweiten Umlaufes. Der dritte Umlauf hat eine Entfernung von mehr als $120000 \mathrm{~km}$ durchlaufen.

Es sei bemerkt, daß solche mehrfachen Erdumläufe nur unter besonders günstigen ionosphärischen Verhältnissen auf den Filmstreifen festgestellt wurden und meistens vor dem Eintreten ionosphärischer Störungen $^{5} \mathrm{zu}$ verzeichnen waren. Über $\mathrm{zu}$ erwartende ionosphärische Stürme, die oft für mehrere Tage Ausfälle bei der gesamten Kurzwellenübertragung zur Folge haben und die von erdmagnetischen Störungen begleitet werden, konnten hierdurch Voraussagen getroffen werden. Gleichzeitig mit den Störungen auftretende Nordlichter wurden oftmals von Frederikshavn aus beobachtet.

5 S. S. Kirby, N. Smith u. T. R. Gilliland, The nature of the ionosphere storm, Physic. Rev. 54, 234 [1938]. 


\section{f) Rechnerische Bestimmung des $t_{u}$-Wertes}

Wenn die Großkreis-Entfernung zwischen Sender und Empfänger bekannt ist und die Laufzeitdifferenz $\mathrm{zw}$ ischen direktem und indirektem Zeichen durch Messungen bestimmt werden kann, kann der $t_{u}$-Wert für jeden Fall berechnet werden, indem man die oben abgeleitete Formel nach $t_{u}$ auflöst und die entsprechenden Werte einsetzt.

Diese Berechnungen wurden für 785 Werte durchgeführt und ergaben $t_{u}=0,137767$ sec als Mittelwert, während bei 218 gemessenen Erdumläufen $t_{u}=0,137788 \mathrm{sec}$ gefunden wurde. Dies entspricht einem Zeitunterschied von $0,000021 \mathrm{sec}$ oder von $0,015 \%$.

\section{Theoretischer Teil}

Betrachtungen über die v.Schmidtsche Kopfwellentheorie

Prof. Dr. Oswald v. Schmidt verstarb am 28. März 1945 in Berlin-Gatow. Der Verfasser dieser Abhandlung hält es für seine Aufgabe, v. Sch midts wesentliche Ideen und Berechnungen weiterzugeben, nach denen eine ionosphärische Kurzwellenausbreitung durch die Kopfwellentheorie bewiesen werden soll.

Abb. 7 zeigt eine solche Gleit- oder Kopfwellen-Ausbreitung. Der vom Sender $S$ nach der Ionosphäre aufsteigende Strahl pflanzt sich in einer ionosphärischen Grenzschicht mit konstanter Geschwindigkeit fort und streut beständig unter einem bestimmten Winkel ${ }^{6}$ aus dieser Schicht Energie nach. der Erdoberfläche ab. Der

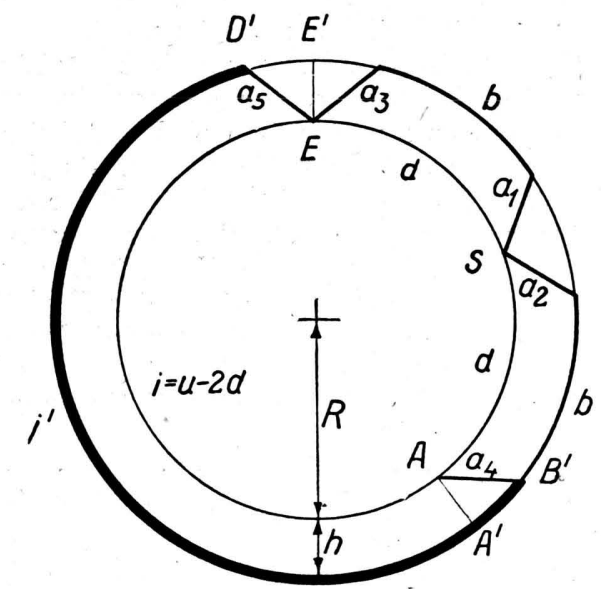

Abb. 7. Gleitwellenausbreitung um die Erde. Direkter Weg: $a_{1}+b+a_{3}$ Indirekter Weg: $a_{2}+b+i^{\prime}+a_{5}$

ionosphärische Weg: $a_{1}+b+a_{3}$ vom Sender zum Empfänger entspricht dem entgegengesetzten Weg:

6 Von Kutowski u. Schüttlöffel bei Telefunken durchgeführte Messungen über Einfallswinkel bei entfernten Kurzwellensendern im Frequenzbereich zwischen 10 und $20 \mathrm{MHz}$ ergaben Werte zwischen $15^{\circ}$ und $22^{\circ}$. $a_{2}+b+a_{4}$ vom Sender bis zum Punkt $A$. Wenn das direkte Zeichen den Empfänger $E$ erreicht hat, verweilt die nach der entgegengesetzten Richtung verlaufende Gleitwelle in der Nähe von $A^{\prime}$. Sie läuft von dort nach $D^{\prime}$ weiter und erreicht den Empfänger
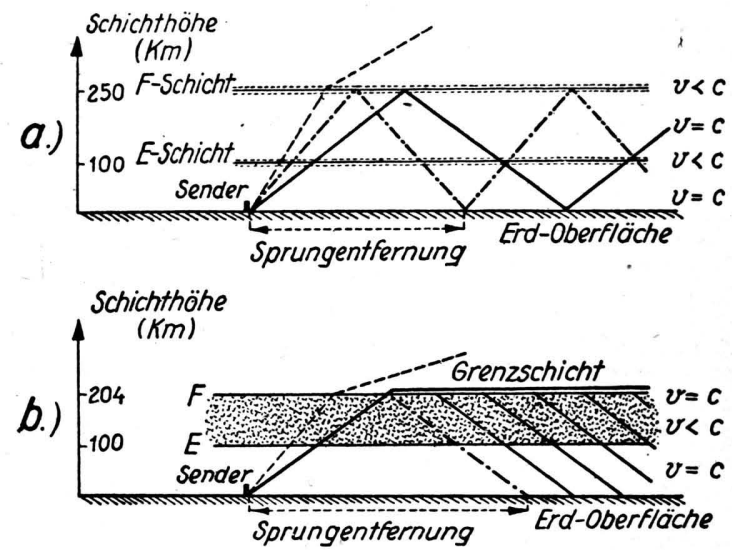

Abb. 8. Kurzwellenausbreitung.

a) Durch mehrfache Reflexionen,

b) durch Gleitwelle nach v. S c h m i d t.

$E$ um die Laufzeit $t_{i}$ später als das direkte Zeichen. Während der Zeit $t_{i}$ wird also der Weg $i^{\prime}$ durchlaufen. Die Tatsache, daß die absteigenden und die aufsteigenden Strahlen $a_{1}, a_{2}, a_{3}$ und $a_{5}$ herausfallen, mag zur Erklärung der überraschenden Genauigkeit bei den gemessenen $t_{i}$ - und $t_{u}$-Werten beitragen. Eine eventuelle Krümmung der Strahłen spielt keine Rolle.

Nimmt man nun an, daß sich die Radiowellen auf dem Kreis vom Radius $R+h$ mit Lichtgeschwindigkeit $c$ fortpflanzen, so liefert die Beziehung:

$$
\frac{R+h}{R}=\frac{c}{(u-2 d) / t_{i}}=\frac{c}{u / t_{u}}
$$

die Möglichkeit der Berechnung der Schichthöhe $\boldsymbol{h}$. Aus 785 guten Vermessungen von Filmstreifen ergab sich als Mittelwert:

$$
w_{i}=\frac{u-2 d}{t_{i}}=290515 \mathrm{~km} / \mathrm{sec}
$$

und aus 218 gut vermeßbaren Erdumläufen:

$$
w_{u}=u / t_{u}=290476 \mathrm{~km} / \mathrm{sec} .
$$

Dies gibt, zusammen mit $R=6370 \mathrm{~km}$ und $c$ $=299776 \mathrm{~km} / \mathrm{sec}$ in die Gleichung

$$
\begin{gathered}
h=R\left(\frac{c}{w_{i}, u}-1\right) \text { eingesetzt: } \\
h=203 \mathrm{~km}\left(w_{i}\right) \text { und } h=204 \mathrm{~km}\left(w_{u}\right) .
\end{gathered}
$$

Diese zwei aus verschiedenartigen Vermessungen stammenden Werte zeigen eine bemerkenswerte Übereinstimmung für die Höhe der ionosphärischen Grenzschicht. 
O. v. S ch m i d befaßte sich mit einer Gegenüberstellung von zwei möglichen Betrachtungen über eine ionosphärische Kurzwellenausbreitung, wie in Abb. 8 a und $8 \mathrm{~b}$ gezeigt wird. Gemäß der Theorie der mehrfachen Reflexionen zwischen Ionosphäre und Erdoberfläche - a - muß innerhalb der reflektierenden $E$ Schicht oder innerhalb der reflektierenden $F$-Schicht die Geschwindigkeit $v$ kleiner sein als die Lichtgeschwindigkeit $c$. Zwischen diesen beiden Schichten und zwischen der $E$-Schicht und der Erdoberfläche ist $v=c$. Die Ausbreitung $-\mathrm{b}-$ ist als eine in einer ionosphärischen Grenzschicht verlaufende, beständig abstrahlende Gleit- oder Kopfwelle dargestellt. Diese Grenzschicht befindet sich $204 \mathrm{~km}$ über der Erdoberfläche. Die Geschwindigkeit $v$ zwischen $E$ - und $F$ Schicht ist kleiner als $c$. In der Grenzschicht und zwischen der Erdoberfläche und der $E$-Schicht ist $v=c$.

Nimmt man nach der Theorie der Radiowellenausbreitung durch mehrfache Zickzack-Reflexionen zwischen Ionosphäre und Erdoberfläche für die Höhe der reflektierenden $F$-Schicht $250 \mathrm{~km}$ an, wie sie auf Grund der Echolotung angenommen wird, so ergibt sich unter Berücksichtigung des $t_{u}$-Wertes von $0,137788 \mathrm{sec}$ ein Abstrahlungswinkel von $3^{0}$ gegen den Horizont. Tatsächlich liegen die von $K u$ tow ski und $\mathrm{Sch}$ üt t 1 öffel gemessenen Werte zwischen 15 und $25^{\circ}$. v. S ch m idt ist der Ansicht, daß bei der Echolotung an den Ionosphärenschichten niemals Entfernungen, wie z. B. Schichthöhen, gemessen werden können, sondern tatsächlich nur Laufzeiten.

Die Tatsache einer überraschend genauen Bestimmung der Entfernungen bei entfernten Kurzwellensendern, zusammen mit anderen experimentellen Befunden, gab v. S c h m i d t die Veranlassung, sie als Wellenausbreitung entlang einer Grenzschicht zwischen zwei Medien zu deuten. Derartige Gleit- oder Kopfwellen hatte v. S chmidt schon auf dem Gebiet der ErdSeismik entdeckt.

\section{Zusammenfassung und Folgerungen}

Das hauptsächliche Ergebnis dieser Untersuchungen offenbart sich in der wichtigen Tatsache, daß Entfernungsbestimmungen bei entfernten Kurzwellensendern im Bereich zwischen 10 und $20 \mathrm{MHz}$ mit großer Genauigkeit möglich sind. Voraussetzung hierfür sind allerdings bestimmte Zustände in der Ionosphäre, die nur zu wenigen Tagesstunden erfüllt sind.

-Wesentliche Punkte der wichtigsten Ergebnisse, denen nur experimentelle Erkenntnisse zugrunde liegen, seien in folgenden Abschnitten kurz zusammengefaßt:

1. Die Wiedergabe der indirekten Zeichen, Umläufe und der mehrfachen Umläufe auf den Filmstreifen ist von seltsamer Klarheit und Schärfe. Es sind dabei keine nennenswerten Verzerrungen durch sogen. Mehr-
weg-Phünomene festzustellen. Indirekte Zeichen und Erdumläufe sind außerdem wesentlich schärfer gezeichnet als die direkten Zeichen. Je größer die durchlaufene Entfernung eines Zeichens ist, desto schärfer ist es auf den Filmen gezeichnet. Größere Verzerrungen der direkten Zeichen traten nur bei solchen Stationen auf, deren Entfernung vom Beobachtungsort kleiner war als $1000 \mathrm{~km}$. In diesen Fällen trat ein Mehrweg-Phänomen in Erscheinung, das durch in kurzen Zeitabständen mit verschiedenen Phasen eintreffende Signale verursacht wird.

2. Vergleiche zwischen den gemessenen Amplituden der direkten Zeichen, indirekten Zeichen, direkten Umläufe, indirekten Umläufe und besonders der mehrfachen Umläufe wurden angestellt. Dabei zeigte sich eine überraschend hohe Feldstärke, die hauptsächlich den Umläufen und mehrfachen Umläufen eigen ist. Amplitudenmessungen zwischen direktem Zeichen und Umlauf bei südamerikanischen Stationen ergaben, daß die Umläufe feldstärkemäßig im Mittelwert 20\% des direkten Zeichens erreichten. Messungen zwischen $\mathrm{Um}$ läufen und mehrfachen Umläufen zeigten Werte bis zu $30 \%$.

3. Zahlreiche Messungen und Untersuchungen über einen Zeitabschnitt von mehr als 3 Jahren ergaben für die Laufzeit des Kurzwellenumlaufes $\boldsymbol{t}_{\boldsymbol{u}}=0,137788 \mathrm{sec}$. Dieser Wert scheint ganz unabhängig zu sein von der Frequenz, ferner von der Tageszeit und der Jahres. zeit. Auch traten keine Änderungen hinsichtlich der Laufzeit während des Verlaufes der 3 Jahre in Erscheinung. Veränderungen der gesamten Kurzwellenausbreitung, die entsprechend einer 11-jährigen Sonnenfleckenperiode eintreten, bewirken eine Verschiebung des Frequenzspektrums, innerhalb dessen die Kurzwellenechos auftreten, und zugleich eine Vergrößerung bzw. eine Verkleinerung der täglichen Echozeiten. Die Erdumlaufszeit bleibt jedoch als eine Konstante bestehen.

4. Nach der v. S c h mi d t schen Theorie ergibt sich für die Umlaufschicht eine Höhe von $203 \mathrm{~km}$ über der Erdoberfläche. Nach der Theorie der Zickzack-Reflexionen ergibt sich bei einem $t_{u}$-Wert von $0,137788 \mathrm{sec}$ und einer Schichthöhe von $250 \mathrm{~km}$ ein Abstrahlungswinkel der Radiowellen von $3^{0}$, während im Durchschnitt Winkel von $20^{\circ}$ gemessen wurden.

Diese experimentell nachgewiesenen Tatsachen stehen in einem bemerkenswerten Widerspruch zu den Ergebnissen der Echolotung an den Ionosphärenschichten bei senkrechter Inzidenz. Die durch die Echolotungen gewonnenen allgemeinen Erkenntnisse wurden bisher auch auf eine Kurzwellenausbreitung über große Entfernungen angewandt. Die daraus entspringende Folgerung, daß diese durch mehrfache Zickzack-Reflexionen zwischen Ionosphäre und Erdoberfläche zustandekommen würde, kann auf Grund der Ergebnisse dieser Abhandlung nicht aufrecht erhalten werden. 